\title{
Alopecia and platelet-derived therapies
}

\author{
John P. Cole ${ }^{1}$, Megan A. Cole ${ }^{1}$, Chiara Insalaco ${ }^{2,3}$, Valerio Cervelli ${ }^{2}$, Pietro Gentile ${ }^{2,4}$ \\ ${ }^{1}$ Cole Hair Transplant Group, Alpharetta, Georgia, USA; ${ }^{2}$ Plastic and Reconstructive Surgery Department, ${ }^{3}$ Institute of Anatomic Pathology, \\ University of Rome Tor Vergata, Rome, Italy; ${ }^{4}$ Plastic and Reconstructive Surgery Department, Catholic University, Tiranna, Albania \\ Contributions: (I) Conception and design: JP Cole; (II) Administrative support: JP Cole, V Cervelli, P Gentile; (III) Provision of study materials or \\ patients: JP Cole, C Insalaco; (IV) Collection and assembly of data: JP Cole, MA Cole, C Insalaco; (V) Data analysis and interpretation: JP Cole, MA \\ Cole; (VI) Manuscript writing: All authors; (VII) Final approval of manuscript: All authors. \\ Correspondence to: John P. Cole, MD. 1045 Powers Place, Alpharetta, GA 30009, USA. Email: john@forhair.com.
}

Background: Platelet rich plasma (PRP) injections have emerged as a promising regenerative therapy for androgenetic alopecia (AGA). To date, injections of both autologous native and activated PRP have been administered to hair loss patients, and positive results have been observed. However, little to no work has yet to be seen wherein PRP treatments are combined with hair restoration surgeries. Furthermore, the PRP activation protocol in the hair restoration setting employs compounds with potentially deleterious side effects, namely thrombin or calcium gluconate. Therefore, the objectives of this work are to evaluate the effectiveness of platelet and platelet-derived products as augmented graft therapies in hair restoration surgeries and to compare the follicular regeneration rate of follicles transplanted in the presence of platelet lysate (PL) versus activated PRP (AA-PRP).

Methods: PL was administered to the frontal scalp of three male AGA patients. Three treatment zones measuring $4 \mathrm{~cm}^{-2}$ were mapped in the midline scalp region of each patient and equal number of follicular grafts were placed in each box along with PL, AA-PRP, or normal saline. The transplanted follicular grafts of a fourth patient were placed solely with PL. Hair checks in which the surface area of hair coverage was quantified were performed at follow-up appointments ranging from 3.5 to 7 months post-surgery. In these appointments, the number of follicular units with hairs measuring $50 \mathrm{~mm}$ or more were counted to determine the percentage of graft hair regeneration. Growth factor (GF) concentrations [vascular endothelial growth factor (VEGF), transforming growth factor beta 1 (TGF- $\beta 1$ ), PDGF-BB, IGF-1] in PL and AA-PRP were also measured for an independent subject set.

Results: Follicular regeneration in transplanted grafts was found to be superior for those placed with PL rather than AA-PRP or saline at all follow-up dates. Specifically, at 3.5 months post-op, $89 \% \pm 9 \%$, $74 \% \pm 7 \%$, and $57 \% \pm 10 \%$ of follicular units had regenerated hair in the PL, AA-PRP, and saline treatment zones, respectively. At 4 months post-op, 99\%, 75\%, and $71 \%$ of follicle regeneration had occurred in the PL, AA-PRP, and saline treatment areas, respectively. Impressively, when PL was injected alone, the patient experienced a $50 \%$ increase in follicular unit density and a $122 \%$ increase in hair density 7 months postinjection. When GF concentrations were measured, PL generated from a 30-min sonication of PRP was found to have significantly higher levels of VEGF, PDGF-BB, and TGF- $\beta 1$ than AA-PRP.

Conclusions: PRP remains a promising hair loss therapy and should be evaluated further for use not only as an independent therapeutic tool, but also as a treatment to augment surgical procedures. PL in particular affords an effective and efficacious therapeutic product given that the lysate may be obtained by mechanical rather than chemical means. Ultrasonic waves provide sufficient energy to rupture platelet cell walls, and centrifugation may be used to separate the lysate from cell fragments prior to delivery.

Keywords: Androgenetic alopecia (AGA); platelet rich plasma (PRP); concentrations

Received: 04 May 2017; Accepted: 19 September 2017; Published: 14 November 2017.

doi: $10.21037 /$ sci.2017.11.01

View this article at: http://dx.doi.org/10.21037/sci.2017.11.01 


\section{Introduction}

Hair loss disorders, such as androgenetic alopecia (AGA), display relatively high rates of occurrence in both men and women. Fifty percent of white men will experience some degree of AGA, also known as male-pattern baldness or MPHL, by age 50 (1), and nearly $50 \%$ of women will experience AGA (female-pattern hair loss, FPHL) over the course of her lifetime (2). Given the prevalence of hair loss in these populations, targeted therapies that reduce the appearance of thinning by delaying, arresting, or reversing the underlying pathology are highly desirable. However, the current selection of pharmacotherapies with US Food and Drug Administration (FDA) approval are limited to oral finasteride, a selective 5 - $\alpha$-reductase inhibitor, and $2 \%$ or $5 \%$ topical minoxidil solutions or foams $(3,4)$, neither of which enable complete hair regrowth, even with persistent use. Moreover, the patient will inevitably face relapse in hair loss if and when the treatment is halted, and undesirable side effects may result from these medications, some of which, such as finasteride-induced impotence, may persist for many years (5).

Alternative cell-based therapies for the treatment of AGA have emerged, of which, platelet rich plasma (PRP) injections appear particularly effective. Although the precise degree to which PRP, improve hair density is difficult to extract from individual studies given the relatively small sample sizes and lack of standardization in follow-up periods, test factors measured, and instruments used to quantify hair count and/or quality, a recent meta-analysis identified PRP as a promising treatment for AGA (6). In practice, autologous PRP (A-PRP) may be applied directly or activated (AA-PRP) prior to delivery. When A-PRP has been injected in its native stage, hair density improvements have ranged from $13 \%$ to $31 \%$ over baseline measurements 3 months following the initial treatment $(7,8)$. Treatment with AA-PRP injections has shown similar hair density improvements 12 to 14 weeks after therapy was begun, with hair densities ranging from $18 \%$ to $29 \%$ above baseline (9-11). However, hair density measurements made 6 months after the start of AA-PRP treatment have shown up to a $56 \%$ improvement over baseline values, although the degree of improvement seems to be influenced by the system used to generate the PRP from whole blood (7).

Since PRP therapies are still in their infancy, and have not been shown to arrest or reverse the hair loss process, many AGA patients turn to surgical intervention to conceal ongoing thinning (12). Within the hair restoration arena, the minimal depth follicular unit isolation technique, particularly when conducted with sharp punches, has proven to be cosmetically effective and affords markedly low transection rates in skilled hands (13). The "minimal depth" ranges from 1.8 to $3.0 \mathrm{~mm}$ below the scalp surface, which places the extraction punch in the deeper dermis or superficial adipose tissue at full stop. Since the average hair follicle extends $4.2 \mathrm{~mm}$ (range, $4-5.1 \mathrm{~mm}$ ) into the scalp (14), the minimal depth extraction approach allows valuable cell populations to remain intact following follicular extraction, namely, epidermal stem cells distal to the sebaceous gland in the bulge region and mesenchymal stem cells in the dermal papilla (15).

In addition to the minimally invasive surgical procedure that conserves vital stem cell populations within the donor region of the scalp, drug therapies that promote wound healing and accelerate regeneration of transplanted hair follicles may also be applied. Products such as hyaluronic acid, extracellular matrix proteins, or PRP may be used either singularly or in an assortment of combinations (16). Uebel et al., for example, have immersed extracted grafts in A-PRP then activated the PRP immediately prior to graft implantation (17). In this work, grafts implanted with AA-PRP displayed significantly higher rates of survival over controls. To date, A-PRP activation, in the context of hair restoration surgeries, has been accomplished via thrombin, calcium chloride, or calcium gluconate solutions as a means to deliver GFs that are either secreted by the platelets or presented on the activated platelet surface (18). However, since each of these products constitute a unique drug therapy with accompanying unwanted or potentially hazardous side effects, an alternative process by which platelet contents may be isolated is warranted for efficacious clinical use. Platelet lysis and accumulation of the released lysate (PL) is one solution, which is accomplished herein via sonication. In this work, the survival rates of hair follicles treated with PL, AA-PRP, or normal saline following transplantation are compared, and the relative growth factor (GF) concentrations for PL and AA-PRP are evaluated.

\section{Methods}

\section{Patient population}

This study enrolled 3 male patients aged 27-55 years who displayed MPHL in Stage 6 as determined by the Norwood-Hamilton classification scale. For each patient, three sets of $2 \mathrm{~cm} \times 2 \mathrm{~cm}$ (or $4 \mathrm{~cm}^{2}$ area) boxes were marked 


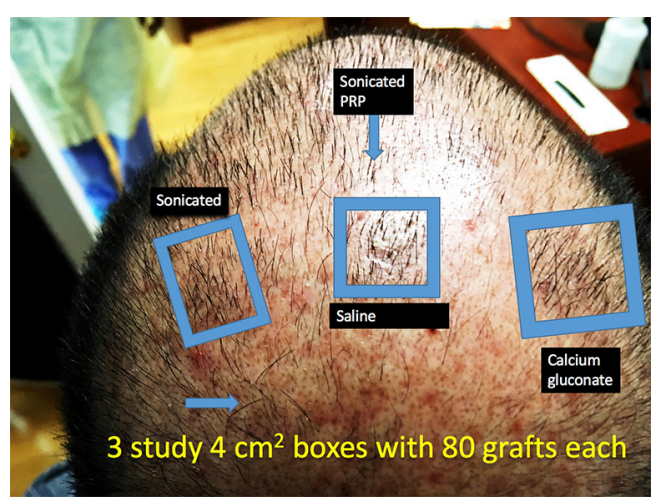

Figure 1 Location of the PL, AA-PRP, and saline treatment zones for Subject A. Eighty grafts were placed in each box, and hair checks were conducted 14 weeks post-surgery. PL, platelet lysate; AA-PRP, autologous activated platelet rich plasma.

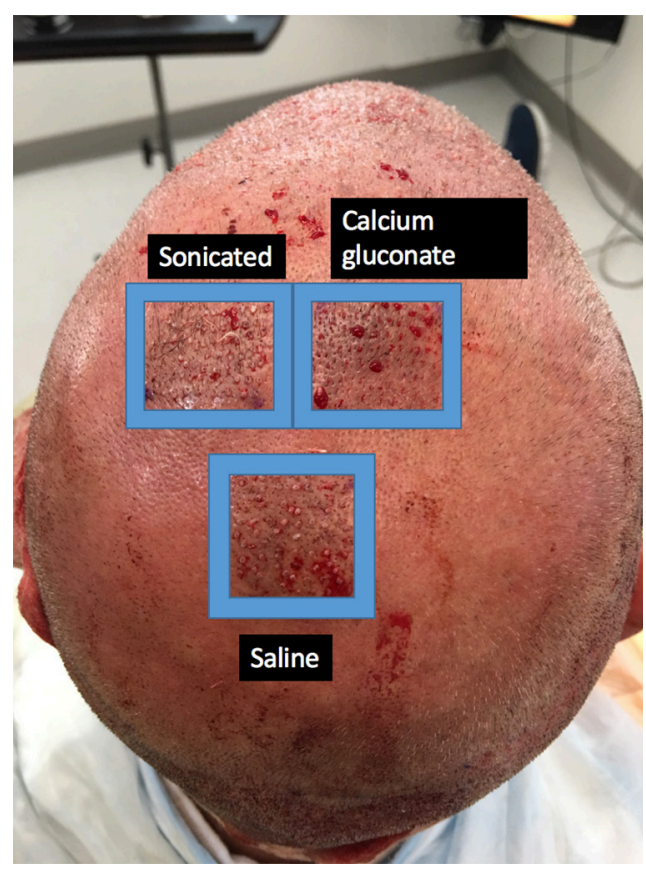

Figure 2 Location of the PL, AA-PRP, and saline treatment zones for Subject B. Eighty grafts were placed in each box, and hair checks were conducted 18 weeks post-surgery. PL, platelet lysate; AA-PRP, autologous activated platelet rich plasma.

in three separate regions of the transplant zone. Follicular unit grafts in conjunction with $1.0 \mathrm{~mL}$ PL, $1.0 \mathrm{~mL}$ calcium gluconate-activated PRP (AA-PRP), or $1.0 \mathrm{~mL}$ normal saline were then placed in each box. The locations of the respective treatment, positive control, and negative control

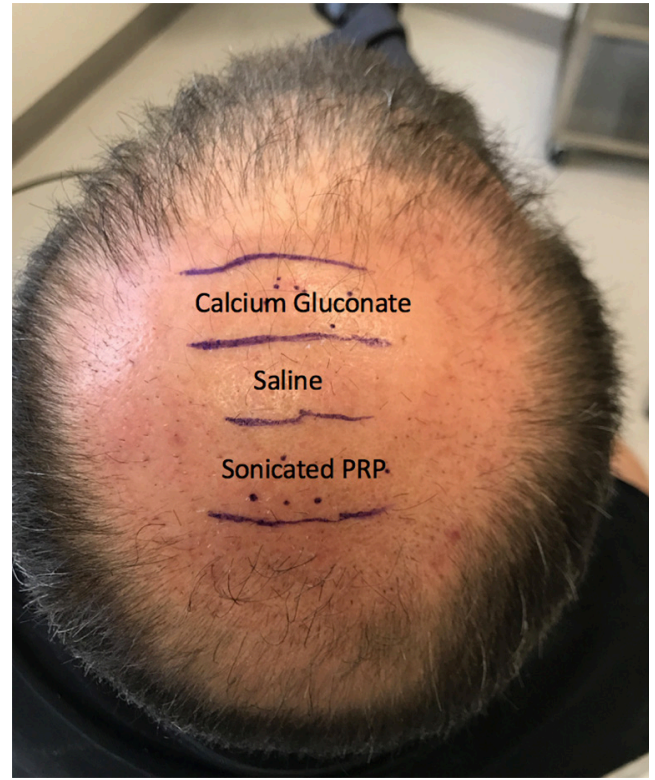

Figure 3 Location of the PL, AA-PRP, and saline treatment zones for Subject C. Forty grafts were placed in each box, and hair checks were conducted 14 weeks post-surgery. PL, platelet lysate; AAPRP, autologous activated platelet rich plasma.

boxes for each patient are outlined in Figures 1-3. Note, however, that the number of hairs within each follicular unit were controlled only in subject C. Only the absolute number of grafts, not the absolute number of hairs was held constant among the three regions in subjects $\mathrm{A}$ and $\mathrm{B}$. The frontal scalp was treated with $0.1 \mathrm{~mL} \mathrm{~cm}^{-2} \mathrm{PL}$ in all patients. A second group of 5 subjects ( 3 male and 2 female aged 20-60 years) with no apparent hair loss was selected solely for analyzing GF concentrations in PL and AA-PRP.

\section{A-PRP preparation, activation, and lysis}

A-PRP was prepared using one of two systems. In the first, Regen Blood Cell Therapy (BCT) tubes were used to prepare A-PRP (15 and $5 \mathrm{~mL}$ per BCT tube) from whole blood $(24 \mathrm{~mL})$ taken from a peripheral vein using sodium citrate as an anticoagulant. The top $2 \mathrm{~mL}$ of A-PRP from each tube was then discarded, giving $9 \mathrm{~mL}$ of A-PRP with a 5 -fold increase in platelet concentration over whole blood. The Regen system A-PRP was activated through the addition of $10 \%(\mathrm{v} / \mathrm{v})$ calcium gluconate, and the resulting AA-PRP was immediately injected at a depth of $3 \mathrm{~mm}$ into the appropriate treatment zone through a $1 \mathrm{~mL}$ Luer lock 


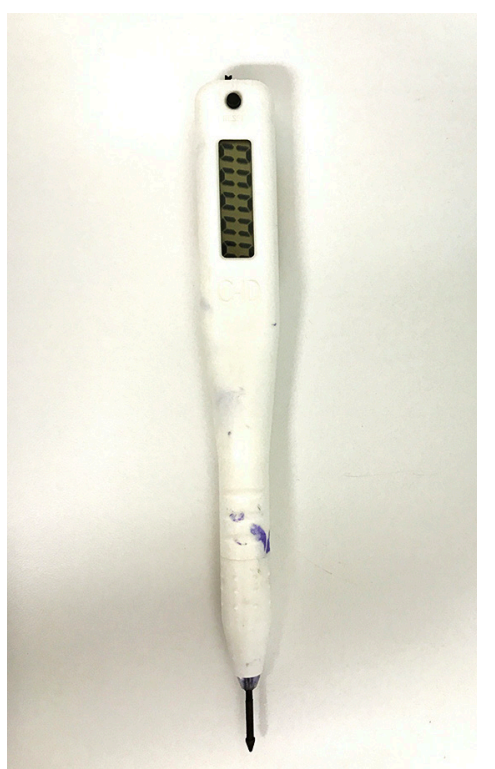

Figure 4 The counting incision device (Cole instruments) used to determine the number of follicles with regenerated hair.

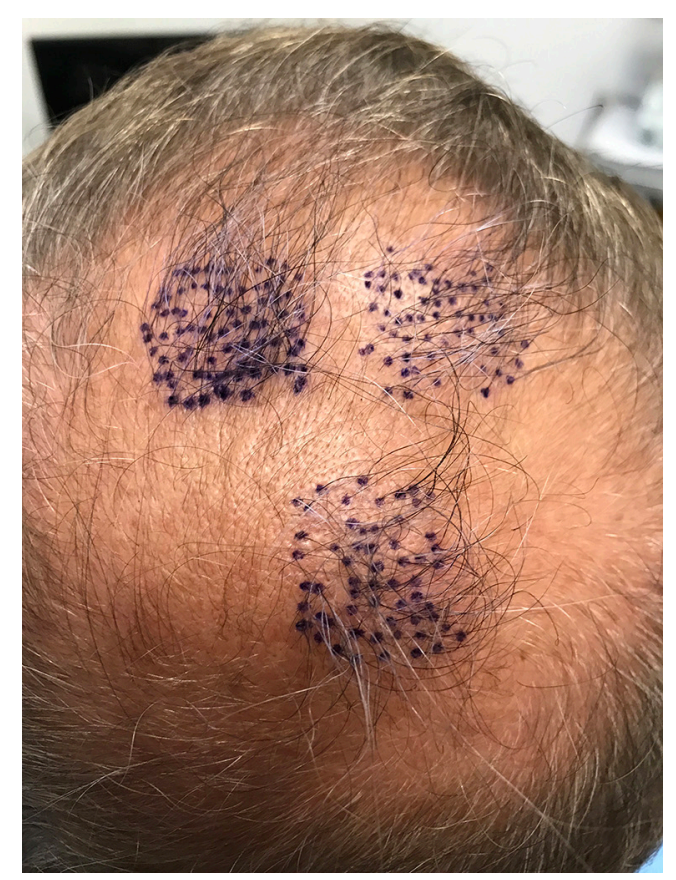

Figure 5 A photograph illustrating the graft recording hair check process. Counted follicular units with hair(s) measuring at least 50 $\mathrm{mm}$ are marked in violet for the treatment regions in Subject B.

syringe equipped with a 25 -gauge needle.

The Arthrex Angel system was also used to prepare
A-PRP (3 mL) from $120 \mathrm{~mL}$ of whole blood with the instrument hematocrit level set to $3 \%$. The A-PRP was then combined with $5 \mathrm{~mL}$ of platelet poor plasma to produce $8 \mathrm{~mL}$ of A-PRP with a 5 -fold increase in platelet concentration over whole blood. A-PRP collected with the Arthrex Angel system was subjected to $30 \mathrm{~s}$ on/30 s off sonication cycles using a Bioruptor Plus sonication device (Diagenode) equipped with six falcon $15 \mathrm{~mL}$ tube holders, each filled with $1.33 \mathrm{~mL}$ of A-PRP and maintained at $4{ }^{\circ} \mathrm{C}$ over the entire sonication period by an accompanying water cooler (115 V minichiller, Diagenode). Total sonication time was set to $30 \mathrm{~min}$ (i.e., $60 \mathrm{on} /$ off cycles). Upon completion of the final cycle, the lysed platelet samples were centrifuged for $10 \mathrm{~min}$ at $1,967 \times \mathrm{g}$, and the supernatant (PL) was recovered and delivered at a depth of $3 \mathrm{~mm}$ into the appropriate treatment zone through a $1 \mathrm{~mL}$ Luer lock syringe equipped with a 25 -gauge needle.

\section{Assessment of hair growth parameters}

For each patient, individual follicular units were counted in each box using a counting incision device (Cole Instruments) loaded with a gentian marker to prevent duplicating the count for any follicular unit and to record a precise number (see Figures 4,5). The number of growing follicular units measuring at least $50 \mathrm{~mm}$ were recorded for each of the three regions of interest, that is, the PL, AAPRP, and saline-treated grafts.

\section{GF quantification}

AA-PRP and PL samples intended for GF concentration testing were prepared as described above. For PL samples, the total sonication time was set to 0 (no treatment), $15 \mathrm{~min}$ (i.e., $30 \mathrm{on} / \mathrm{off}$ cycles), or $30 \mathrm{~min}$ (i.e., $60 \mathrm{on} / \mathrm{off}$ cycles). All samples were centrifuged for $10 \mathrm{~min}$ at $1,967 \times \mathrm{g}$, and the recovered supernatant was stored at $4{ }^{\circ} \mathrm{C}$ prior to testing. The concentrations of four GFs [PDGF-BB, IGF-1, transforming growth factor beta 1 (TGF- $\beta 1$ ), and vascular endothelial growth factor (VEGF)] were measured using commercially-sourced enzyme-linked immunosorbent assay (ELISA) kits (Table 1) in accordance with standard, previously reported protocol (14). Briefly, standards and samples were added to a 96-well microplate pre-coated with an antibody against the target GF. An enzyme-linked polyclonal antibody, specific for the target GF was added in excess and the unbound antibody was rinsed away. A 
substrate solution was then added and color developed in proportion to the quantity of bound GF. After the color development was stopped, the absorbance was measured at $450 \mathrm{~nm}$ using a $\mu$ Quant microplate reader (Bio-Tek). GF concentrations were determined from standard curves with the aid of GraphPad Prism curve-fitting software. For IGF- 1 and TGF- $\beta 1$, the data was linearized by plotting the $\log$ (concentration) versus the $\log (\mathrm{OD} 450)$, and a best fit was found via linear regression analysis. For all others, a nonlinear four-parameter logistic curve fit was performed. For Regen-derived GF measurements, $n=5$. GF measurements for the Arthrex Angel system contained only 2 of the above 5 subjects $(n=2)$.

\section{Statistical analysis}

Platelet GF concentrations are expressed as mean \pm standard error $(\mathrm{n}=5$ or $\mathrm{n}=2)$. Unpaired $t$-tests $(\alpha=0.05)$ were used to determine differences in mean among the baseline GF concentrations in A-PRP derived from the Regen and Arthrex systems. One-way Analysis of Variance (ANOVA) was used to determine statistical significance between data sets $(\alpha=0.05)$. For data sets with significant differences in means, the Tukey test was applied to identify the source(s) of variation

Table 1 Commercially-sourced ELISA kits used to quantify platelet growth factors

\begin{tabular}{ll}
\hline Target protein & ELISA assay kit specifications \\
\hline PDGF-BB & Cat \# EHPDGFB, Thermo Scientific \\
VEGF & Cat \# KGH011, Novex Life Technologies \\
IGF-1 & Cat \# DG100, R\&D Systems \\
TGF- $\beta 1$ & Cat \# KAC1688, Invitrogen Life Technologies \\
\hline
\end{tabular}

ELISA, enzyme-linked immunosorbent assay; VEGF, vascular endothelial growth factor; TGF- $\beta 1$, transforming growth factor beta 1.

\section{Results}

The number of visible follicular units within the PL, AA-PRP, and control boxes at follow-up, in addition to the number of follicular units placed within each box during the surgical procedure, are provided in Table 2 for the three primary participants of this study. Transplanted follicular unit growth was highest in those regions treated with PL, where $89 \% \pm 9 \%$ and $99 \%$ of follicular units were visible at 14 and 18 weeks, respectively. Although the AA-PRP region appeared more successful than the control at 14 weeks with $74 \% \pm 7 \%$ versus $57 \% \pm 10 \%$ of transplanted grafts visible, there was little disparity between these two groups at 18 weeks ( $75 \%$ versus $72 \%$ for AA-PRP and saline treatment zones, respectively).

For comparative purposes, the entire scalp of a single subject (Subject D, 44, Norwood Five) was treated exclusively with PL $(10 \mathrm{~mL})$. Four tattoos were drawn on the mid-scalp, and hair checks were performed on these regions at 28 weeks post-treatment. At this time, the patient demonstrated an increase in visible follicular unit density and hair density from starting values of $60 \mathrm{FU} \mathrm{cm}^{-2}$, and 90 hair $\mathrm{cm}^{-2}$ to $90 \mathrm{FU} \mathrm{cm}$, and 200 hair $\mathrm{cm}^{-2}$. Before and after photos for this patient are shown in Figure 6. As a control, 18 grafts were placed into a scar and treated only with saline. At 16 weeks, only 10 of these grafts were visible.

Concentrations of IGF- 1 , TGF- $\beta 1$, PDGF-BB, and VEGF for intact and lysed Arthrex-derived PRP and native and activated Regen-derived PRP are provided in Table 3. No significant difference was observed between native PRP collected via Regen BCT or the Arthrex Angel system for any of the GFs evaluated in this study. Overall, the highest concentrations were obtained from Arthrex PRP samples that underwent a $30 \mathrm{~min}$ sonication. One notable exception was the IGF-1 value, as no significant difference in means was found for this $\mathrm{GF}(\mathrm{P}=0.98)$. For the remaining three $\mathrm{GFs}$

Table 2 Follicular units placed and counted in the PL, AA-PRP, and control regions at follow-up for the primary subjects

\begin{tabular}{llllll}
\hline \multirow{2}{*}{ Subject } & Follicular units placed per $4 \mathrm{~cm}^{2}$ & Follow-up (weeks) & \multicolumn{3}{l}{ Follicular units (no.) } \\
\cline { 4 - 6 } & & & 14 & PL & AA-PRP \\
\hline A & 80 & 18 & 64 & 54 & 53 \\
B & 80 & 14 & 79 & 60 & 57 \\
C & 40 & 14 & 39 & 32 & 19 \\
\hline
\end{tabular}

PL, platelet lysate; AA-PRP, autologous activated platelet rich plasma. 


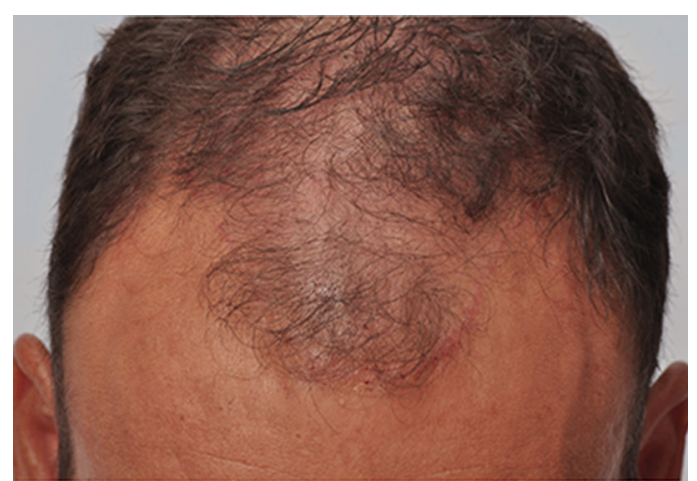

Before

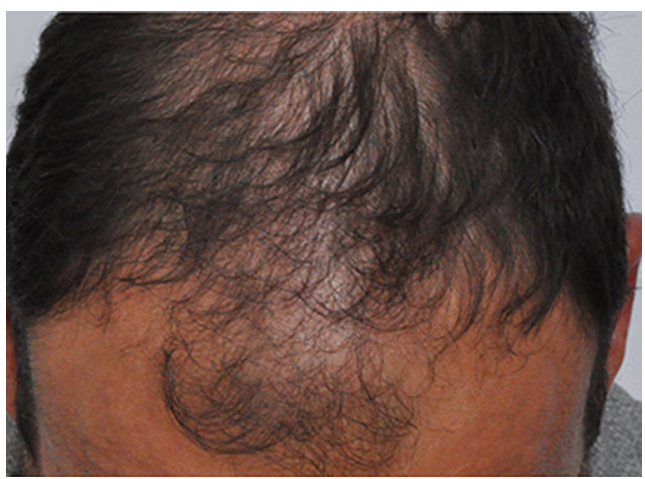

Six months after sonicated PRP

Figure 6 Before and after photos of Subject D. Hair check in the after photo was performed 28 weeks post-injection.

Table 3 PL, PRP, and AA-PRP growth factor concentrations

\begin{tabular}{|c|c|c|c|c|c|}
\hline Growth factor & \multicolumn{2}{|c|}{ Regen BCT } & \multicolumn{3}{|c|}{ Arthrex angel system } \\
\hline IGF-1 (ng mL ${ }^{-1}$ ) & $130 \pm 20$ & $140 \pm 20$ & $150 \pm 40$ & $140 \pm 40$ & $150 \pm 40$ \\
\hline TGF- $\beta 1$ (ng mL ${ }^{-1}$ ) & $11 \pm 2$ & $15 \pm 3$ & $12 \pm 1$ & $50 \pm 8$ & $93 \pm 30$ \\
\hline PDGF-BB & $1.2 \pm 0.3$ & $4 \pm 2$ & $1.1 \pm 0.6$ & $19 \pm 0$ & $24 \pm 2$ \\
\hline
\end{tabular}

${ }^{\dagger}, \mathrm{PL}$ generated from 15 min total sonication time; ${ }^{\ddagger}, \mathrm{PL}$ generated from 30 min total sonication time. PL, platelet lysate; AA-PRP, autologous activated platelet rich plasma; BCT, Blood Cell Therapy; TGF- $\beta 1$, transforming growth factor beta 1 ; VEGF, vascular endothelial growth factor.

(i.e., TGF- $\beta 1$, VEGF, and PDGF-BB), a 30-min sonication produced significantly higher concentrations than did activation with calcium gluconate, which was not significantly different from native Regen PRP with respect to any GF.

\section{Discussion}

The results of this work show that transplanted grafts resume growth faster in the presence of $\mathrm{PL}$ than treatment with AA-PRP or saline. However, for Subject A, it was noted that the quality of grafts in the Regen-derived AAPRP region was superior to those in the Arthrex-derived PL region upon initial placement and in the follow-up evaluation at 14 weeks. Even though the coverage was aesthetically better in the AA-PRP box, the number of grafts growing was higher in the PL box, a phenomenon likely attributable to the grafts in the Regen box coincidentally containing a greater number of hairs per follicular unit than those placed in the PL box. Nevertheless, the faster rate of regrowth for grafts placed in the presence of PL cannot be disputed. Indeed, the singular, beneficial influence of $\mathrm{PL}$ in hair regeneration is best illustrated in Subject D, as this patient was treated exclusively with PL over his entire recipient region; no graft transplantations were performed. As previously noted, the frontal regions of Subjects A, B, and $\mathrm{C}$, were treated with PL, and since vascular flow from the frontal scalp to adjacent regions of the scalp cannot be blocked, the potential for follicular regeneration within the AA-PRP or saline boxes to be positively influenced by PL injected in the frontal scalp could not be avoided entirely. Importantly, the locations of the three test boxes in the recipient region of the scalp were shuffled in an effort to account for any contamination of the results.

A plausible hypothesis for the superior follicular regeneration rate of PL-treated grafts over AA-PRPtreated grafts may be drawn based on the relative GF concentrations in PL and AA-PRP, respectively. With the previously noted exception of IGF-1, which was statistically 
equivalent for all test groups, PL contained significantly higher GF concentrations than AA-PRP. As suggested by their names, GFs promote cell growth through several pathways. VEGF, for example, promotes angiogenesis, which establishes a conduit for nutrient delivery and waste removal (19), and TGF- $\beta 1$ prevents basal keratinocytes from reaching terminal differentiation which ensures the proliferative potential of this cell population is retained (20). When dermal papilla cells are cultured in the presence of activated PRP, higher concentrations of molecules linked to hair growth are measured with respect to controls. Specifically, higher levels of $\beta$-catenin, the protein in the Wnt pathway that is responsible for stimulating hair follicle development $(21,22)$, have been observed (23). Thus, it is reasonable to expect grafts placed with higher concentrations of platelet-derived GFs to regenerate hair follicles sooner than those placed with lower concentrations of the same molecules.

Given that PL and AA-PRP are derived from identical sources (i.e., platelet concentrated whole blood), one may question why PL contains markedly higher levels of most GFs. The answer likely stems from the mechanism by which platelets relinquish GFs in the respective processes. When platelets are activated by exposure to calcium gluconate, GFs are released from the $\alpha$-granules and glycoprotein $\mathrm{IIb} / \mathrm{III}$, a receptor able to bind fibrinogen, is translocated to the cell membrane (18). Binding of fibrinogen to glycoprotein receptors on adjacent platelets causes them to adhere to one another, and coagulation ensues. Ultimately, a platelet plug is formed, within which a high concentration of GFs is trapped. The rate at which these bioactive proteins can reach surrounding tissues to initiate their respective pathways becomes diffusion- and mesh sizelimited. Small proteins should be released from the plug faster than large proteins which may require some degree of plug degradation before they are released. Alternatively, when platelets are lysed, the contents of $\alpha$-particles are released without aggregation, as the cell walls are no longer intact. In terms of a therapy tool, the limiting factor for concentrating GFs becomes the percentage of platelets that are lysed in a given sonication session.

The relative GF concentrations measured in this study indicate that molecular size does influence recovery in a method dependent manner. IGF-1 $(7.6 \mathrm{kDa})$, a protein that is substantially smaller than TGF- $\beta 1(\sim 25 \mathrm{kDa})$, PDGFBB $(\sim 30 \mathrm{kDa})$ or VEGF ( 38 kDa) (24-26), was present in statistically equivalent concentrations for all PRP treatments. The remaining GFs, which are very similar in size, exhibited a six- to eight-fold greater concentration in PL originating from a 30-min sonication period than in AA-PRP. Conceivably, these larger proteins were not readily expelled out of the platelet plug upon centrifugation owing to pores within the plug being too small.

The immediate and positive influence of a bolus GF delivery from PL on follicular regeneration has been discussed above; nevertheless, given the upward trend in follicular unit density and hair density when PL is administered as a stand-alone therapy, one may conclude (albeit with limited confidence, $\mathrm{n}=1$, Subject $\mathrm{D}$ ) that secondary factors are at play. We hypothesize that PL may afford activation of additional platelets and/or initiation of relevant hair stimulation pathways more effectively than AA-PRP since the platelet cell membranes are removed. In $\mathrm{PL}$, molecules that participate in the clotting cascade (i.e., ADP and ATP from dense granules) or that are released to activate additional platelets (i.e., thromboxane A2 from $\alpha$-granules) are free to diffuse into the surrounding tissue without being bound by platelets within the venous blood draw that were concentrated into the PL precursor, A-PRP. Since the precise cellular mechanisms encompassing the beneficial nature of PL in hair replacement surgeries has yet to be identified, the authors recommend that this field be more thoroughly examined given the promising, side effectlimited nature of the therapy.

\section{Acknowledgements}

This work was supported by a grant from the International Society of Hair Restoration Surgery.

\section{Footnote}

Conflicts of Interest: The authors have no conflicts of interest to declare.

Ethical Statement: Informed consent was obtained from all study patients.

\section{References}

1. Ellis JA, Sinclair R, Harrap SB. Androgenetic alopecia: pathogenesis and potential for therapy. Expert Rev Mol Med 2002;4:1-11.

2. Rogers NE, Avram MR. Medical treatments for male and female pattern hair loss. J Am Acad Dermatol 2008;59:54766; quiz 567-8. 
3. Rousso DE, Kim SW. A review of medical and surgical treatment options for androgenetic alopecia. JAMA Facial Plast Surg 2014;16:444-50.

4. Schweiger ES, Boychenko O, Bernstein RM. Update on the pathogenesis, genetics and medical treatment of patterned hair loss. J Drugs Dermatol 2010;9:1412-9.

5. Olsen EA, Weiner MS. Topical minoxidil in male pattern baldness: effects of discontinuation of treatment. J Am Acad Dermatol 1987;17:97-101.

6. Gupta AK, Carviel JL. Meta-analysis of efficacy of platelet-rich plasma therapy for androgenetic alopecia. J Dermatolog Treat 2017;28:55-8.

7. Gentile P, Cole JP, Cole MA, et al. Evaluation of NotActivated and Activated PRP in Hair Loss Treatment: Role of Growth Factor and Cytokine Concentrations Obtained by Different Collection Systems. Int J Mol Sci 2017;18. pii: E408.

8. Takikawa M, Nakamura S, Nakamura S, et al. Enhanced effect of platelet-rich plasma containing a new carrier on hair growth. Dermatol Surg 2011;37:1721-9.

9. Gentile P, Garcovich S, Bielli A, et al. The Effect of Platelet-Rich Plasma in Hair Regrowth: A Randomized Placebo-Controlled Trial. Stem Cells Transl Med 2015;4:1317-23.

10. Gkini MA, Kouskoukis AE, Tripsianis G, et al. Study of platelet-rich plasma injections in the treatment of androgenetic alopecia through an one-year period. J Cutan Aesthet Surg 2014;7:213-9.

11. Cervelli V, Garcovich S, Bielli A, et al. The effect of autologous activated platelet rich plasma (AAPRP) injection on pattern hair loss: clinical and histomorphometric evaluation. Biomed Res Int 2014;2014:760709.

12. Bouhanna P, Bouhanna E. Hair Transplantation for Aesthetic Surgery of the Scalp and Body Hair. In: Bouhanna P, Bouhanna E, editors. Alopecias: Diagnosis and Treatments. Boca Raton: CRC Press-Taylor \& Francis Group, 2016:163-88.

13. Cole JP. Status of Individual Follicular Group Harvesting. Hair Transplant Forum Int 2009;19:20.

14. Jimenez F, Izeta A, Poblet E. Morphometric analysis of the human scalp hair follicle: practical implications for

doi: $10.21037 /$ sci.2017.11.01

Cite this article as: Cole JP, Cole MA, Insalaco C, Cervelli V, Gentile P. Alopecia and platelet-derived therapies. Stem Cell Investig 2017;4:88. the hair transplant surgeon and hair regeneration studies. Dermatol Surg 2011;37:58-64.

15. Zhang H, Tasnim F, Ying JY, et al. The impact of extracellular matrix coatings on the performance of human renal cells applied in bioartificial kidneys. Biomaterials 2009;30:2899-911.

16. Rose PT. Hair restoration surgery: challenges and solutions. Clin Cosmet Investig Dermatol 2015;8:361-70.

17. Uebel CO, da Silva JB, Cantarelli D, et al. The role of platelet plasma growth factors in male pattern baldness surgery. Plast Reconstr Surg 2006;118:1458-66; discussion 1467.

18. Nurden AT. Platelets, inflammation and tissue regeneration. Thromb Haemost 2011;105 Suppl 1:S13-33.

19. Pierce GF, Mustoe TA, Lingelbach J, et al. Plateletderived growth factor and transforming growth factor-beta enhance tissue repair activities by unique mechanisms. J Cell Biol 1989;109:429-40.

20. Shipley GD, Pittelkow MR, Wille JJ Jr, et al. Reversible inhibition of normal human prokeratinocyte proliferation by type beta transforming growth factor-growth inhibitor in serum-free medium. Cancer Res 1986;46:2068-71.

21. Lichtenberger BM, Mastrogiannaki M, Watt FM. Epidermal $\beta$-catenin activation remodels the dermis via paracrine signalling to distinct fibroblast lineages. Nat Commun 2016;7:10537.

22. Andl T, Reddy ST, Gaddapara T, et al. WNT signals are required for the initiation of hair follicle development. Dev Cell 2002;2:643-53.

23. Li ZJ, Choi HI, Choi DK, et al. Autologous platelet-rich plasma: a potential therapeutic tool for promoting hair growth. Dermatol Surg 2012;38:1040-6.

24. Rinderknecht $\mathrm{E}$, Humbel RE. The amino acid sequence of human insulin-like growth factor I and its structural homology with proinsulin. J Biol Chem 1978;253:2769-76.

25. Neufeld G, Cohen T, Gengrinovitch S, et al. Vascular endothelial growth factor (VEGF) and its receptors. FASEB J 1999;13:9-22.

26. Assoian RK, Komoriya A, Meyers CA, et al. Transforming growth factor-beta in human platelets. Identification of a major storage site, purification, and characterization. J Biol Chem 1983;258:7155-60. 